\title{
ALGAS FITOPLANCTÓNICAS EN LA EVALUACIÓN DE LA CALIDAD DEL AGUA DE SISTEMAS LÓTICOS EN EL NOROESTE ARGENTINO
}

\author{
Claudia Seeligmann ${ }^{(1)}$, Beatriz C. Tracanna ${ }^{(1-3)}$, Silvia Martínez De Marco ${ }^{(2)}$ y Sara Isasmendi ${ }^{(1)}$ \\ (1) Instituto de Limnología del Noroeste Argentino (ILINOA) de la Facultad de Ciencias Naturales e IML, \\ Universidad Nacional de Tucumán; (2)Fundación Miguel Lillo; ${ }^{(3)}$ CONICET. Miguel Lillo 205, (4000) San Miguel \\ de Tucumán, Tucumán, Argentina. E-mail: ilinoa@csnat.unt.edu.ar
}

\section{RESUMEN}

En este trabajo se ha caracterizado la calidad de las aguas de los ríos Salî́, Gastona, Medina y Marapa, tributarios del embalse Río Hondo (Noroeste Argentino), durante el período 1992-1995, empleando a las algas como indicadoras. El embalse recibe de los cuatro ríos desechos de las industrias azucareras, alcoholeras, papeleras y citrícolas, entre otras, que aportan el mayor número de contaminantes en valores de DBO,. Esto se ha puesto de manifiesto principalmente en época de zafra, cuando el Salí y el Gastona presentan anoxia, registros de hasta $1200 \mathrm{mg} / \mathrm{l} \mathrm{de} \mathrm{DBO,,} 405 \mathrm{mg} / \mathrm{l}$ de nitratos y $2 \mathrm{mg} / \mathrm{l} \mathrm{de}$ amonio. En los ríos Medina y Marapa los valores de oxígeno disuelto han sido mayores a $4 \mathrm{mg} / \mathrm{l}$, la DBO, no ha superado los $52 \mathrm{mg} / \mathrm{l}$ y los nitratos y amonio han alcanzado registros de 154 y $1.04 \mathrm{mg} / \mathrm{l}$, respectivamente.

Se han identificado un total de 124 taxones algales. En los ríos Salí y Gastona, en términos de riqueza y densidad, el grupo más importante es el de las diatomeas seguidas por las clorofitas, mientras que en el Medina y en el Marapa son más abundantes las algas verdes. Los euglénidos en los cuatro tributarios, han alcanzado hasta un $40 \%$ del total en el año 1995. La diversidad ha sido, en general, mayor hacia la prezafra, aunque se observaron altos registros para algunos periodos contaminados.

El índice de Pantle y Buck se ha relacionado mejor con la química del agua al modificar el índice sapróbico (s) de las siguientes especies: Aulacoseira granulata (Ehrenberg) Simonsen, Chlamydomonas incerta Pascher, Closterium acutum var. variabile (Lemmermann) Krieger, Cocconeis placentula var. euglypta (Ehrenberg) Cleve, Cyclotella meneghiniana Kützing, Cymbella helvetica Kützing, Diatoma vulgare Bory, Euglena acus Ehrenberg, E. oxyuris Schmarda. E. proxima Dangeard, Gomphonema augur Ehrenberg, G. parvulum (Kützing) Kützing, Gyrosigma acuminatum (Kützing) Rabenhorst, Hantzschia amphioxys (Ehrenberg) Grunow, Melosira varians Agardh, Navicula cuspidata Kützing, $N$. pupula Kützing, Nitzschia acicularis (Kützing) W. Smith, N. linearis (Agardh) W. Smith, N. palea (Kützing) W. Smith, N. sigmoidea (Nitzsch) W. Smith, Oscillatoria chalybea Mertens, Pinnularia maior (Kützing) Rabenhorst y Uva casinöensis Playfair.

El ACP de las muestras basado en la abundancia del fitoplancton, ha determinado cambios en la composición y en la abundancia de las especies dominantes. El factor 1 establece diferencias de los ríos Salí y Gastona de Marapa y Medina y el factor 2 refleja una distribución temporal de los taxones separando los períodos 92-93 de 94-95.

Palabras clave: fitoplancton, calidad del agua, sistemas lóticos, Noroeste Argentino.

\begin{abstract}
The water quality of rivers Sali, Gastona, Medina and Marapa, tributaries of Dam Rio Hondo (North-West of Argentina) was determined during the period 1992-1995 using alga as indicators.

The dam receives waste through thefour riversfrom the sugar; alcohol, paper und citric industries, representing the largest contributors as measured by $B O D_{5}$ values. During the sugar cane cropping period, rivers Sali and Gastona presented anoxia with valuesof $1200 \mathrm{mg} / \mathrm{l}$ of $B O D$,, $405 \mathrm{mg} / \mathrm{l}$ of nitrate and $2 \mathrm{mg} / \mathrm{l}$ of ammonia. The dissolved oxygen in the rivers Medina and Marapa was higher than $4 \mathrm{mg} / \mathrm{l}$. BOD, did not exceed $52 \mathrm{mg} / \mathrm{l}$. Nitrate and ammonia reached 154 and $1.04 \mathrm{mg} / \mathrm{l}$ respectively. A total of 124 taxa offreshwater algae were identified. The diatoms were the most richest group in terms of species and densityfollowed by the chlorophytes in rivers Sali and Gastona, while the green algae were the most abundant in rivers Medina und Marapa. The euglenoids reached $40 \%$ of the total in the,fourtributaries in 1995. The diversity was higher before the sugar cane cropping period. However; high recordsfor some contaminated periods were also observed.

Pantle and Buck's index could be improved in their water chemistry adjustement by changing the saprobic index value attached to thefollowing species: Aulacoseira granulata (Ehrenberg)Simonsen, Chlamydomonas incerta Pascher, Closterium acutum var. variabile (Lemmermann) Krieger, Cocconeis placentula var: euglypta (Ehrenberg) Cleve, Cyclotella meneghiniana
\end{abstract}

Limnetica 20(1): 123-133 (2001)

C Asociación Española de Limnología, Madrid. Spain. ISSN: 0213-8409 
Kützing, Cymbella helvetica Kützing, Diatoma vulgare Bory, Euglena acus Ehrenberg, E. oxyuris Schmarda, E. proxima Dangeard, Gomphonema augur Ehrenberg, G. parvulum (Kützing) Kützing, Gyrosigma acuminatum (Kützing) Rabenhorst, Hantzschia amphioxyc (Ehrenberg) Grunow, Melosira varians Agurdh, Navicula cuspidata Kützing, N. pupula Kützing, Nitzschia acicularis (Kïtzing) W. Smith, N. linearis (Agardh)W. Smith, N. palea (Kützing) W. Smith, N. sigmoidea (Nitzsch)W. Smith, Oscillatoria chalybea Mertens, Pinnularia maior (Kützing)Rabenhorst and Uva casinöensis Playfair.

The PCA of samples based on the abundance of phytoplankton, revealed changes in the dominance and abundance of species. Factor one in the PCA established differences between rivers Gastona and Sali und rivers Marapa and Medinu; andfactor two showed changes in tuxu in periods $92-93$ and $94-95$ periods.

Key words: Phytoplankton, water quality. lotic systems, Northwest of Argentina.

\section{INTRODUCCIÓN}

Los estudios limnológicos del embalse Río Hondo (Argentina) se iniciaron en el año 1992 y generaron resultados fisicoquímicos y del fitoplancton de la zona limnética del embalse y de los ríos Salí y Gastona (Locascio et al., 1997; Romero et al., 1994, 1997; Mirande y Tracanna, 1995; Mirande et al., 1996; Seeligmann et al., 1996; Tracanna et al., 1994, 1996a, b, 2000). La represa fue construida para la atenuación de crecidas, riego, provisión de agua potable y energía hidroeléctrica, deporte y turismo. Recibe desechos de las industrias azucareras, alcoholeras, papeleras y citrícolas, entre otras y vertidos cloacales, que aportan el mayor volumen de contaminantes en valores de demanda bioquímica de oxígeno (DBO,). El estado trófico del embalse fue caracterizado como eu-politrófico para la zona de la cola con presencia de euglénidos en época de zafra y oligomesotrófico para la olla en los restantes períodos del año (Locascio et al., 1997; Tracanna et al., 1999). En 1995 Microcystis aeruginosa y Anabaena flos-aquae se manifestaron por primera vez como floración (Tracanna et al., 1996a).

Desde hace algunas décadas es reconocida la importancia del uso de las algas y en especial de las diatomeas como indicadores biológicos, a través de métodos ecológicos (riqueza específica, diversidad, índices de saprobicidad y análisis multivariados). En Argentina, los monitoreos de ambientes acuáticos se basaron principalmente en la valoración físicoquímica y bacteriológica del agua. Con respecto a la comunidad fitoplanctónica los estudios estuvieron centrados en rela- cionar sus cambios espaciales y temporales con respecto a las variables abióticas. En esta línea podemos mencionar entre otros a Bonetto et al., 1982, 1983; del Giorgio et al., 1991; García de Emiliani, 1980, 1986, 1990, 1997; Luque y Martínez de Fabricius, 2000; Mirande et al., 1999; O'Farrell, 1993, 1994; O'Farrell \& Izaguirre, 1994; Tracanna et al., 1999; Zalocar de Domitrovic y Vallejos, 1982; Zalocar de Domitrovic, 1990. Sin embargo, son escasos los resultados que evidenciaron la eficacia del uso de algas planctónicas como bioindicadoras para evaluar la calidad del agua de los ríos (Seeligmann, 1999; Seeligmann et al., 1999, Tangorra et al., 1998 y otros).

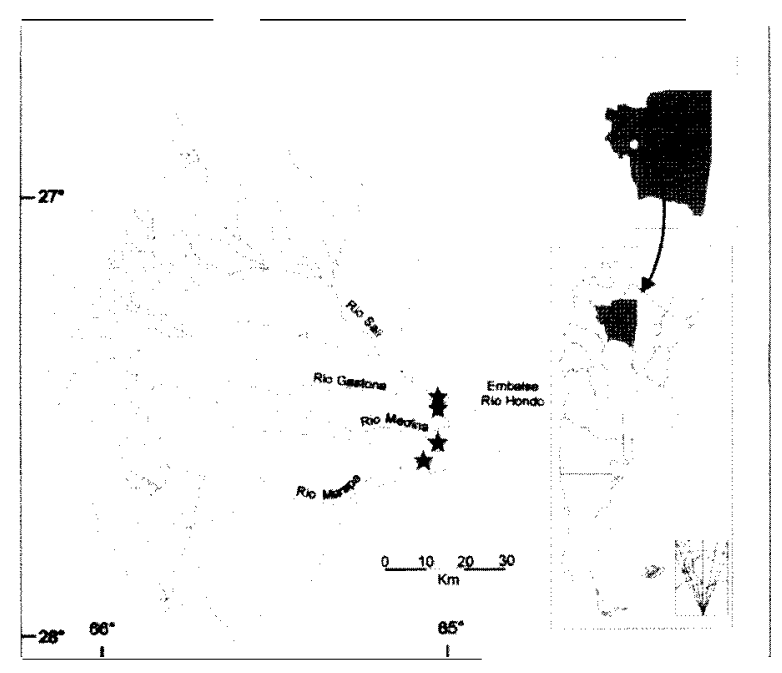

Figura 1. Localización de los lugares de muestreo: ríos Salí (RS), Gastona (RG), Medina (RMe) y Marapa (RMa). Location of sampling stations: rivers Salí (RS), Gastona $(R G)$, Medina $(R M e)$ and Marapa (RMa). 
El objetivo de este trabajo es emplear al fitoplancton como indicador a través de su composición y abundancia, para caracterizar el agua de las desembocaduras de los tributarios del embalse Río Hondo durante los años 1992-1995. Para ello se creó una lista de especies con índices sapróbicos regionales.

\section{MATERIAL Y MÉTODOS}

El embalse Río Hondo (Noroeste Argentino) está ubicado a 27" 30' $\mathbf{S}$ y 65" 00' W y recibe cuatro tributarios: Salí, Gastona, Medina y Marapa (Fig. 1) (Locascio et al., 1997).

Los muestreos fueron realizados dos veces por año, durante el período 1992-1995, en las desembocaduras de los cuatro ríos, teniendo en cuenta la actividad azucarera que se extiende de julio a noviembre. Los análisis químicos se hicieron de acuerdo a APHA (1992). Las muestras cualitativas se obtuvieron con red de plancton de $25 \mu \mathrm{m}$ de malla y las cuantitativas con botellas de plástico de $1.500 \mathrm{~cm}^{3}$, ambas fijadas "in situ" con formaldehído al $4 \%$. Los recuentos algales se efectuaron mediante microscopio invertido, aceptándose un error no mayor al $20 \%$.

Para la evaluación de la calidad del agua en relación al fitoplancton se empleó el índice sapróbico (SI) de Pantle y Buck (195.5) a través de la fórmula: SI $=\Sigma(\mathrm{s} . \mathrm{h}) / \Sigma \mathrm{h}$, donde h corresponde a la densidad algal y s al índice sapróbico de Sládecek (1973) o al estimado según el comportamiento regional de las especies en base a la limnosaprobicidad (Lange-Bertalot, 1979) (Tabla 1). Asimismo, la diversidad específica fue calculada según el índice de Margalef (1958).

El análisis de Componentes Principales (ACP) se efectuó con las densidades algales (valores normalizados y estandarizados) y se trabajó con la matriz de correlación. Cabe señalar que de las 124 especies determinadas en las muestras cualitativas, sólo 56 fueron observadas en los recuentos, lo que indica que las restantes se encontraron en bajas concentraciones. Del total de especies cuantificadas se seleccionaron 27 (Tabla 1) teniendo en cuenta la frecuencia $(\geq 5$ muestras) y/o abundancia ( $>2$ ind/I). Los datos se procesaron mediante el paquete estadístico NTSYS (1991).

También, se realizó un análisis de correlación (coeficiente de Pearson) entre los dos primeros componentes del ACP, las variables químicas (nitritos, nitratos, amonio, oxígeno disuelto, demanda bioquímica de oxígeno) y la densidad de las especies seleccionadas de las 23 muestras estudiadas, haciendo uso del programa SPSS (1992). En este trabajo se comentan sólo aquellas correlaciones que resultaron significativas $(\mathrm{p}<$ 0.05 у $\mathrm{p}<0.01)$.

\section{RESULTADOS}

En época de zafra, los ríos Salí y Gastona presentaron anoxia y registros de hasta $1200 \mathrm{mg} / \mathrm{l}$ de DBO, $405 \mathrm{mg} / \mathrm{l}$ de nitratos y $2 \mathrm{mg} / \mathrm{l} \mathrm{de}$ amonio. En los ríos Medina y Marapa los valores de oxígeno disuelto fueron mayores a $4 \mathrm{mg} / \mathrm{l}$, la DBO, no superó los $52 \mathrm{mg} / 1$ y los nitratos y amonios alcanzaron 154 y $1.04 \mathrm{mg} / \mathrm{l}$, respectivamente. Según la concentración de estos parámetros y en términos de limnosaprobicidad, los dos primeros ríos fueron clasificados como polisaprobios y los dos últimos como a-mesosaprobios a polisaprobios en invierno y primavera respectivamente, mientras que los cuatro ríos resultaron b-mesosaprobios para verano y otoño.

Se identificaron un total de 124 taxones de algas y el grupo más importante fue el de las diatomeas con 49 especies, seguidas por las algas verdes con 42. Se pudo generalizar que en los ríos Salí y Gastona la mayor densidad algal estuvo representada por las Bacillariophyceae, sobresaliendo Cyclotella meneghiniana Kützing, Nitzschia palea (Kützing) W. Smith y Synedra ulna (Nitzsch) Ehrenberg, mientras que en los ríos Medina y Marapa fueron más abundantes las Chlorophyta especialmente Chlamydomonas incerta Pascher y Pandorina morum (Müller) Bory. Las Cyanophyta y Dinophyceae no tuvieron importancia numérica, sin embargo, las Euglenophyta en los cuatro ríos, alcanzaron hasta un $40 \%$ del total en el año 1995 (Tabla 1, 
Tabla 1. Valores promedios de abundancia e índice sapróbico (s) de las especies seleccionadas (ind/l) (1992 - 1995) de los ríos Salí, Gastona, Medina y Marapa ( $\mathrm{s}=$ índice sapróbico regional; $\mathrm{P}=$ prezafra; $\mathrm{Z}=$ zafra). Average abundance ojselected species (ind/l) (1992 - 1995) of rivers Salí, Gastona, Medinu and Marapa rivers ( $s=$ regional saprobic index $\boldsymbol{P}=$ before sugar cane cropping; $Z=$ sugar cane cropping).

\begin{tabular}{|c|c|c|c|c|c|c|c|c|c|}
\hline & \multirow[t]{2}{*}{$\mathbf{s}$} & \multicolumn{2}{|c|}{ Río Salí } & \multicolumn{2}{|c|}{ Río Gastona } & \multicolumn{2}{|c|}{ Río Medina } & \multicolumn{2}{|c|}{ Río Marapa } \\
\hline & & $\mathbf{P}$ & $\mathrm{Z}$ & $\mathbf{P}$ & $\mathbf{Z}$ & $\mathbf{P}$ & $\mathbf{Z}$ & $\mathbf{P}$ & $\mathrm{Z}$ \\
\hline \multicolumn{10}{|l|}{ Cyanophyta } \\
\hline Oscillatoria chalybea Mertens & 4 & 37 & 705 & 13 & 35 & 0 & 0 & 24 & 0 \\
\hline \multicolumn{10}{|l|}{ Chlorophyta } \\
\hline Chlamydomonas incerta Pascher & 2 & 0 & 0 & 0 & 0 & 49135 & 398101 & 0 & 747067 \\
\hline $\begin{array}{l}\text { Closterium acutum var. variabile } \\
\text { (Lemmermann) Krieger }\end{array}$ & 3.5 & 73 & 295 & 20 & 165 & 860 & 775 & 93 & 690 \\
\hline Pandorina morum (Müller) Bory & 2 & 13 & 50 & 180 & 30 & 75855 & 43388 & 105 & 10920 \\
\hline $\begin{array}{l}\text { Scenedesmus quadricauda (Turpin) } \\
\text { Brébisson }\end{array}$ & 2 & 33 & 0 & 187 & 50 & 0 & 0 & 118 & 0 \\
\hline Uva casinoensis Playfair & 4 & 0 & 1870 & 0 & 280 & 0 & 2873 & 140 & 5747 \\
\hline \multicolumn{10}{|l|}{ Bacillariophyceae } \\
\hline $\begin{array}{l}\text { Aulacoseira granulata (Ehrenberg) } \\
\text { Simonsen }\end{array}$ & 3.5 & 847 & 2185 & 260 & 815 & 1720 & 7182 & 538 & 12643 \\
\hline $\begin{array}{l}\text { Cocconeis placentula var. euglypta } \\
\text { (Ehrenberg) Cleve }\end{array}$ & 2 & 67 & 550 & 60 & 60 & 1725 & 863 & 60 & 0 \\
\hline Cyclotella meneghiniana Kützing & 2 & 23600 & 775 & 6220 & 630 & 8620 & 4310 & 3425 & 0 \\
\hline Cymbella helvetica Kützing & 2 & 80 & 65 & 467 & 90 & 0 & 0 & 278 & 0 \\
\hline Diatoma vulgare Bory & 3.5 & 33 & 300 & 7 & 85 & 0 & 0 & 46 & 0 \\
\hline Gomphonema augur Ehrenberg & 4 & 147 & 300 & o & 0 & 0 & 0 & 0 & 0 \\
\hline G. parvulum (Kützing) Kützing & 4 & 40 & 265 & 40 & 140 & 0 & 0 & 90 & 0 \\
\hline $\begin{array}{l}\text { Gyrosigma acuminatum (Kützing) } \\
\text { Rabenhorst }\end{array}$ & 2 & 213 & 60 & 20 & 25 & 0 & 9193 & 23 & 18387 \\
\hline $\begin{array}{l}\text { Hantzschia amphioxys } \\
\text { (Ehrenberg) Grunow }\end{array}$ & 4 & 107 & 430 & 93 & 0 & 0 & 0 & 47 & 0 \\
\hline Melosira varians Agardh & 4 & 93 & 815 & 67 & 30 & 0 & 0 & 48 & 0 \\
\hline Navicula cuspidata Kützing & 3.5 & 1120 & 585 & 220 & 605 & 0 & 0 & 413 & 0 \\
\hline N. pupula Kützing & 4 & 67 & 520 & 60 & 460 & 0 & 2873 & 260 & 5747 \\
\hline Nitzschia acicularis (Kützing) & & & & & & & & & \\
\hline W. Smith & 4 & 487 & 105 & 87 & 55 & 0 & 0 & 71 & 0 \\
\hline Nitzschia linearis (Agardh) W. Smith & 4 & 40 & 95 & 20 & 35 & 0 & 0 & 28 & 0 \\
\hline N. palea (Kützing) W. Smith & 4 & 3973 & 6035 & 880 & 1205 & 0 & 3448 & 1043 & 6897 \\
\hline N. sigmoidea (Nitzsch) W. Smith & 4 & 13 & 245 & 27 & 0 & 0 & 0 & 13 & 0 \\
\hline Pinnularia maior (Kützing) & & & & & & & & & \\
\hline Rabenhorst & 3.5 & 327 & 260 & 107 & 30 & 0 & 0 & 68 & 0 \\
\hline Synedra ulna (Nitzsch) Ehrenberg & 2 & 5587 & 3285 & 407 & 105 & 0 & 573 & 256 & 1147 \\
\hline Euglenophyceae & & & & & & & & & \\
\hline Euglena acus Ehrenberg & 4 & 13 & 650 & 13 & 370 & 0 & 287 & 192 & 573 \\
\hline E. oxyuris Schmarda & 3.5 & 13 & 20 & 0 & 195 & 0 & 172 & 98 & 343 \\
\hline E. proxima Dangeard & 4 & 27 & 23070 & 173 & 1460 & 0 & 14080 & 817 & 28160 \\
\hline
\end{tabular}




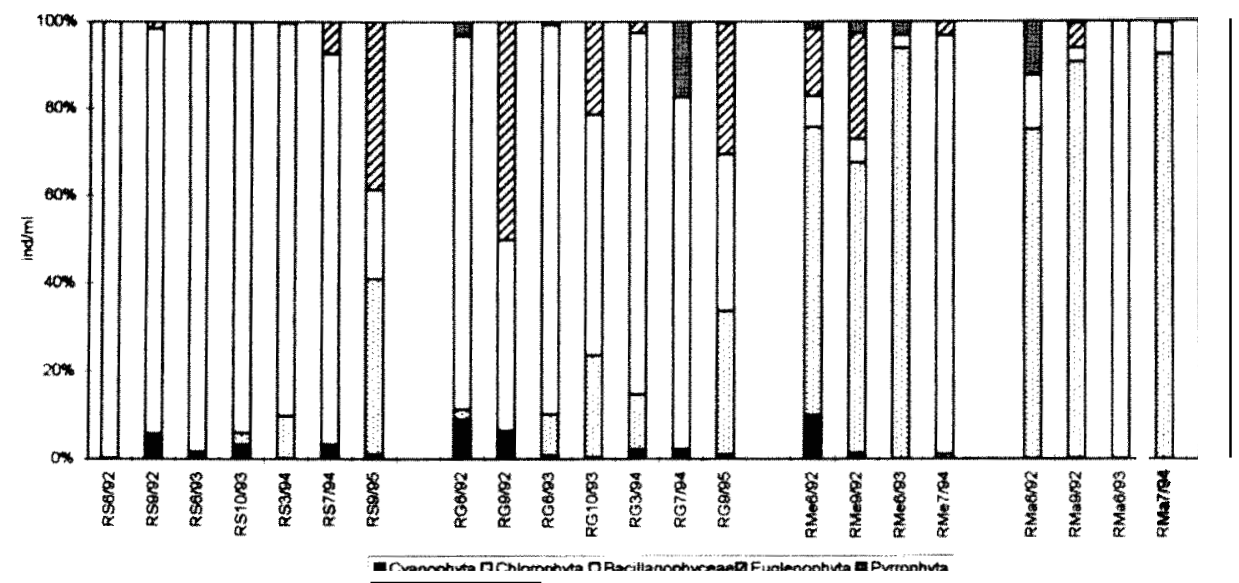

Figura 2. Abundancia relativa del fitoplancton de los ríos Salí, Gastona, Medina y Marapa. Phytoplankton relative abundance of the Rivers Salí, Gastonu, Medina and Marapa
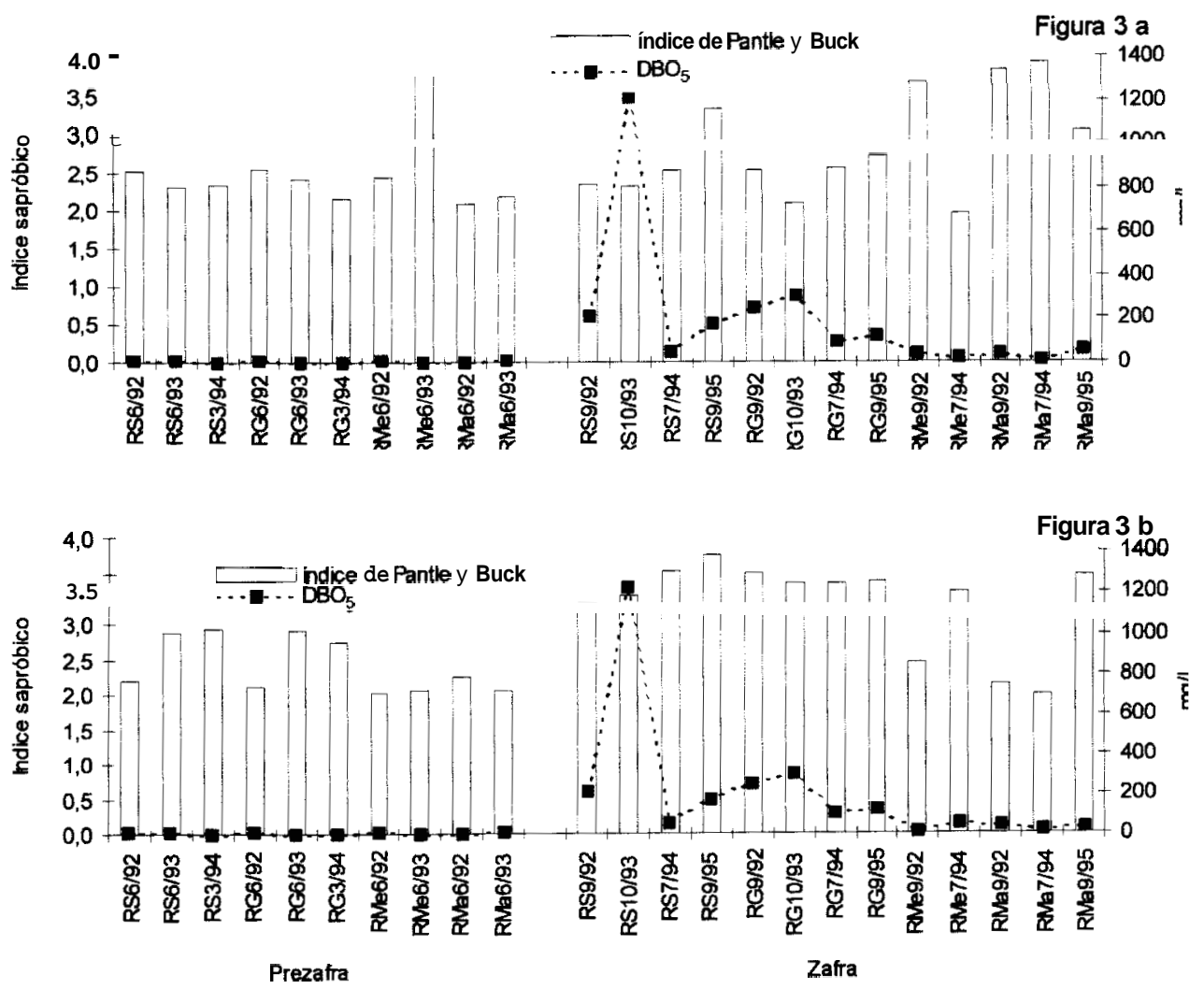

Figura 3. a) Índice de Pantle y Buck utilizando el índice sapróbico de Sládecek (1973) y su relación con la DBO, en los ríos Salí, Gastona, Medina y Marapa. b). Índice de Pantle y Buck en base al comportamiento regional de las especies y su relación con la DBO, en los ríos Salí. Gastona, Medina y Marapa. a) Pantle and Buck's index using Slúdecek's saprobic index (1973)and its relationship with the BOD, of rivers Sali, Gastona, Medina and Marapa. b) Pantle and Buck's index based on the regional behaviour of species and their relationship with the $B O D$, of'rivers Salí, Gastona, Medina and Marapa. 
Fig. 2). El índice de Pantle y Buck (1955) (Fig. 3 a), utilizando el índice sapróbico dado por Sládecek (1973) varió entre 2.1-3.3 en los ríos Salí y Gastona, para la época de zafra, y entre 1.9-3.9 en Medina y Marapa. Durante la prezafra fue en general, inferior a 2.6, excepto en el río Medina que alcanzó 3.9. Los altos registros de saprobicidad no siempre fueron acompañados por bajos valores de oxígeno disuelto. Sin embargo, este índice se ajustó mejor a la química del agua al adjudicarle a las distintas especies un valor en relación al comportamiento regional (Tabla 1, Fig. 3 b).

Según el criterio de clasificación de las especies en sensibles, tolerantes y muy tolerantes a la contaminación orgánica (Lange-Bertalot, 1979) y de acuerdo al rango sapróbico (Sládecek, 1973) (Fig. 4), se observó en general un predominio de tolerantes para la zona $\beta$-mesosapróbica y un mayor desarrollo de muy tolerantes en las zonas $\alpha$-mesosapróbicas a polisapróbicas. Algunas especies citadas como sensibles estuvieron presentes tanto en zonas $\beta$-mesosapróbicas como polisapróbicas.
El índice de diversidad (Fig. 5) fue mayor, en general, hacia la prezafra, período en que los registros de DBO, no superaron los $9.5 \mathrm{mg} / \mathrm{l}$. Sin embargo, es importante destacar que en algunos meses más contaminados también se observaron valores de diversidad de hasta 3.8.

Como resultado del análisis de correlación (Tabla 2) se determinó que Comphonema augur Ehrenberg, Hantzschia amphioxys (Ehrenberg) Grunow, Nitzschia. sigmoidea (Nitzsch) W. Smith y Oscillatoria chalybea Mertens presentaron con la DBO, una correlación positiva, siendo explicada la variación de estas especies en más de un $60 \%\left(\mathrm{r}^{2}>0.60, \mathrm{p}<0.01\right)$ por este parámetro. Por otro lado, Nitzschia sigmoidea y Oscillatoria chalybea tuvieron una correlación negativa en relación al oxígeno disuelto a un $\mathrm{p}<0.05$, con un $\mathrm{r}^{2}$ de 0.31 y 0.22 , respectivamente. Asimismo, Euglena proxima Dangeard, Cymbella helvetica Kützing y Gomphonema parvulum (Kützing) Kützing estuvieron positivamente correlacionadas $\left(\mathrm{r}^{2}>0.50, \mathrm{p}<0.01\right)$ con las concentraciones de nitratos y Euglena acus Ehrenberg con los registros de amonio $\left(r^{2}=0.26, p<0.05\right)$.

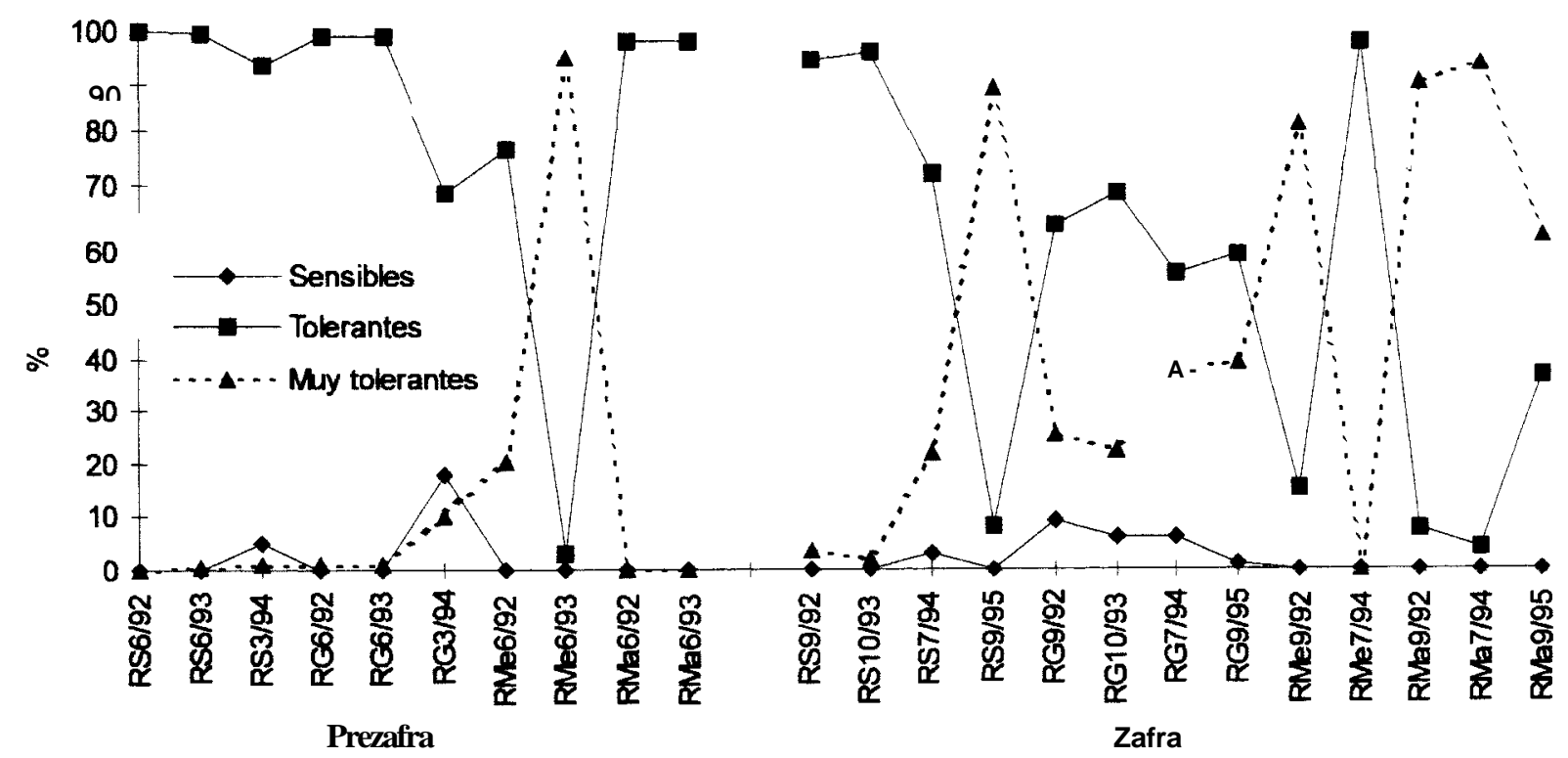

Figura 4. Variaciones de la abundancia relativa de las especies sensibles, tolerantes y muy tolerantes a la polución de los ríos Salí, Gastona, Medina y Marapa. Changes in the relative abundance of pollution sensitive, tolerant and very tolerant species of rivers Sali, Gastona, Medina and Marapa. 
Tabla 2. Matriz de correlación entre las variables seleccionadas (DBO,: demanda bioquímica de oxígeno, OD: oxígeno disuelto, $\mathrm{NO}_{3}{ }_{3}^{-}$: nitrato, $\mathrm{NH}_{4}{ }^{+}$: amonio, Osc: Oscillatoria chalybea, Cyh: Cymbella helvetica, Ga: Gomphonema augur, Gp: Gomphonema parvulum, Ha: Hantzschia amphioxys, Mv: Melosira varians, Ns: Nitzschia sigmoidea, Np: Nitzschic palea, Pm: Pinnularia muior, Ea: Euglena acus, Ep: Euglena proxima, $\mathrm{F} 1$ : factor $1,{ }^{* *}: \mathrm{p}<0.01,{ }^{*}: \mathrm{p}<0.05, \mathrm{n}: 23$ ). Correlation matrix between selected variables (BOD ${ }_{5}$ : bioche mical oxygen demand, $\mathrm{DO}$ : dissolved oxygen, $\mathrm{NO}_{3}$ : nitrate, $\mathrm{NH}_{4}{ }^{+}$: ammonia, $\mathrm{Osc}$ : Oscillatoria chalybea, $\mathrm{Cvh}$ : Cymbella helvetica, Ga: Gomphonema augur, $\mathrm{Gp}$ : Gomphonema parvulum, Hat Hantzschia amphioxys, $M v$ : Melosira varians, $N s$ : Nitzschia sigmoidea, $N p$ : Nitzschia palea, Pm: Pinnularia maior, Ea: Euglena acus, Ep: Euglena proxima, FI: factor $1, * *: p<0.01, *: p<0.05, n: 23)$

\begin{tabular}{|c|c|c|c|c|c|c|c|c|c|c|c|c|c|c|c|c|}
\hline & DBO, & OD & NO, & $\mathrm{NH}^{+}{ }_{4}$ & Osc & Cyh & $\mathbf{G a}$ & Gp & Ha & Mv & Ns & Np & Pm & $\mathbf{E a}$ & $\mathbf{E p}$ & F 1 \\
\hline $\begin{array}{l}\text { DBO, } \\
\text { OD }\end{array}$ & $\begin{array}{r}1 \\
-0.66 " ' "\end{array}$ & 1 & & & & & & & & & & & & & & \\
\hline $\mathrm{NO}_{3}^{-}$ & -0.01 & 0.19 & 1 & & & & & & & & & & & & & \\
\hline $\mathrm{NH}_{4}^{+}$ & $0.57 "$ & -0.42 & 0.33 & 1 & & & & & & & & & & & & \\
\hline $\mathrm{Osc}^{4}$ & $0.95 " "$ & $-0.47^{\prime \prime}$ & -0.11 & 0.48 & & & & & & & & & & & & \\
\hline Cyh & -0.11 & 0.09 & $0.97 * *$ & 0.08 & -0.09 & 1 & & & & & & & & & & \\
\hline $\mathrm{Ga}$ & $0.86^{* *}$ & -0.32 & -0.09 & 0.47 & $0.94 * *$ & -0.09 & 1 & & & & & & & & & \\
\hline $\mathrm{Gp}$ & -0.2 & 0.11 & $0.99^{* *}$ & 0.16 & -0.12 & $0.24-$ & -0.13 & 1 & & & & & & & & \\
\hline $\mathrm{Ha}$ & $0.89 * *$ & -0.36 & -0.09 & 0.48 & $0.97 * *$ & 0.06 & $0.97 * *$ & -0.08 & 1 & & & & & & & \\
\hline $\mathrm{Mv}$ & $0.67 * *$ & -0.13 & -0.04 & 0.48 & $0.74 * *$ & -0.12 & $0.69 " "$ & -0.14 & $0.71 " "$ & 1 & & & & & & \\
\hline Ns & $0.87 * *$ & $-0.56 * *$ & -0.15 & 0.32 & $0.90^{* *}$ & -0.12 & $0.80 " '$ & -0.12 & $0.84 * *$ & $* 0.64 * *$ & 1 & & & & & \\
\hline $\mathrm{Np}$ & $0.52 *$ & -0.27 & -0.01 & 0.36 & $0.56 " "$ & -0.07 & $0.57 " '$ & 0.02 & $0.57^{* *}$ & 0.39 & $0.48^{\prime \prime}$ & 1 & & & & \\
\hline $\mathrm{Pm}$ & $0.52 *$ & -0.29 & -0.22 & 0.18 & $0.60 * *$ & -0.19 & $0.70 " "$ & -0.26 & $0.60 * *$ & 0.34 & $0.69 * *$ & 0.35 & 1 & & & \\
\hline $\mathrm{Ea}$ & 0.14 & -0.41 & 0.30 & $0.51 *$ & -0.01 & $-0.06-$ & -0.06 & 0.23 & -0.07 & -0.10 & -0.09 & -0.17 & -0.17 & 1 & & \\
\hline Ep & -0.2 & 0.04 & $0.71 " " '$ & 0.24 & -0.01 & $0-$ & -0.11 & $0.57 * *$ & -0.12 & -0.11 & -0.14 & 0.21 & -0.22 & 0.40 & I & \\
\hline F 1 & $0.96^{* *}$ & $-0.57 *$ & -0.11 & 0.48 & $0.97 * *$ & -0.12 & $0.95 " '$ & -0.13 & $0.95 * *$ & $0.96^{* * *}$ & $0.94 * *$ & $0.59 *$ & $0.82 * *$ & -0.08 & -0.21 & 1 \\
\hline
\end{tabular}




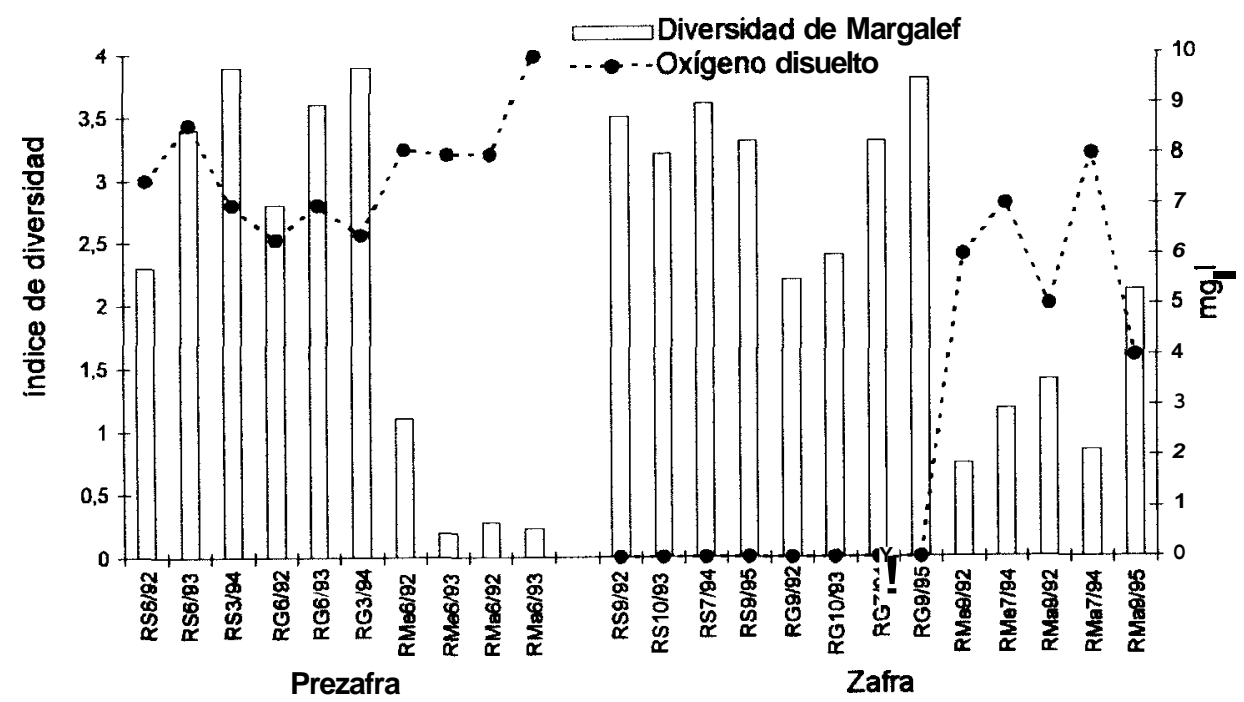

Figura 5. Índice de diversidad de Margalef y su relación con el oxígeno disuelto de los ríos Salí, Gastona, Medina y Marapa. Margalef diversity index and its relation with dissolved oxigen of the Salí, Gastona, Medina and Marapa rivers. Margalef s diversity index and their relationship with the oxygen concentration in rivers Sali, Gastona, Medina and Marapa.

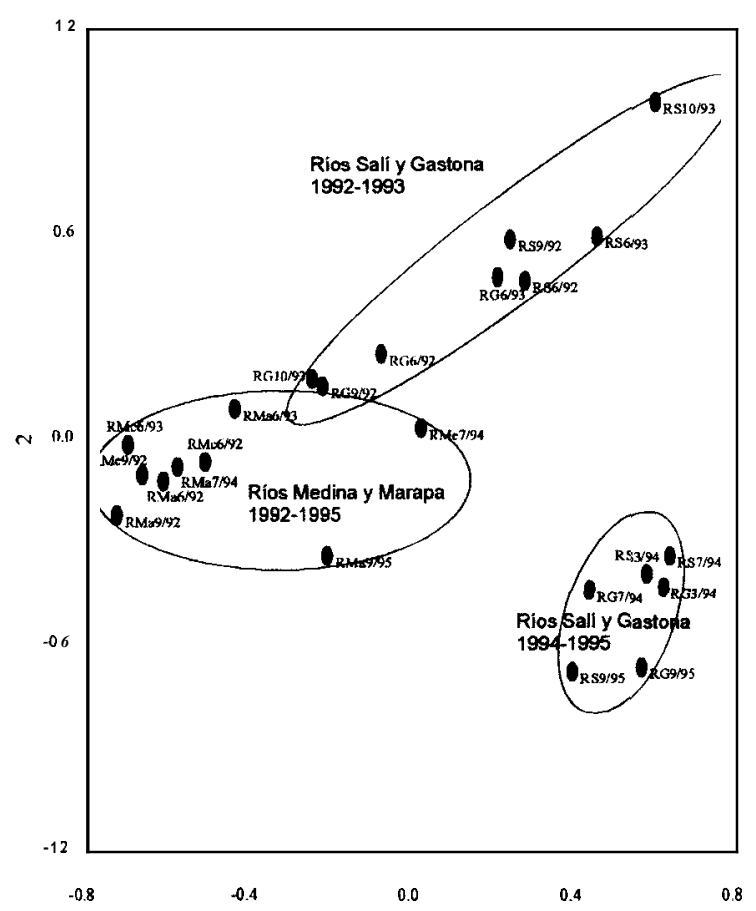

Figura 6. Representación de los dos primeros ejes del análisis de Componentes Principales (ACP) de las muestras basado en la abundancia del fitoplancton. Biplot of the two first axes of the Principal Component analysis (PCA) of samples based in the abundance of phytoplankton.
El ACP (Fig. 6) demostró cambios en la composición y en la abundancia de las 27 especies seleccionadas. Los dos primeros ejes acumularon un 45 $\%$ de la varianza total. El factor 1 (25\% de la varianza) estableció diferencias entre los ríos Salí y Gastona con respecto a Marapa y Medina, a través de Nitzschia palea, N. acicularis (Kützing) W. Smith, Navicula cuspidata Kützing y Chlamydomonas incerta. Asimismo, este factor presentó una correlación positiva con la DBO, $\left(\mathrm{r}^{2}=0.92, \mathrm{p}<\right.$ $0.01)$ y negativa con el oxígeno disuelto $\left(r^{2}=-0.32\right.$, $\mathrm{p}<0.05)$. Esta relación, indica la existencia de un gradiente de contaminación desde los ríos Medina y Marapa hacia Gastona y Salí. El factor $2(20 \%$ de la varianza) reflejó una distribución temporal de los taxones separando los períodos 92-93 de 94-95 por medio de Oscillatoria chalybea, Navicula pupula Kützing, Gomphonema parvulum y Nitzschia linearis (Agardh) W. Smith.

\section{DISCUSIÓN Y CONCLUSIONES}

Existen distintos criterios sobre la efectividad de la aplicación de los índices de saprobicidad y 
diversidad específica para evaluar la calidad del agua (Gómez, 1998, 1999; Lobo et al., 1995). De acuerdo a nuestros resultados la diversidad se ajustó mejor a los cambios que tuvieron lugar en relación a la carga orgánica. Sin embargo, no se podría utilizar este índice para caracterizarla debido a que se obtuvieron altos registros tanto en aguas limpias como contaminadas.

La escasa relación que presentó el índice de Pantle y Buck con las zonas de saprobicidad cuando se lo estimó utilizando el índice sapróbico de Sládecek, se debería a que especies citadas como sensibles, presentaron un crecimiento vigoroso en aguas severamente contaminadas, con registros de DBO, de hasta $172 \mathrm{mg} / 1$. Es por ello, que en base al comportamiento de las especies en el área de muestreo se propone una modificación del índice sapróbico (s) de: Aulacoseira granulata (Ehrenberg) Simonsen, Chlamydomonas incerta, Closterium acutum var. variabile (Lemmermann) Krieger, Cocconeis placentula var. euglypta (Ehrenberg) Cleve, Cyclotella meneghiniana, Cymbella helvetica, Diatoma vulgare Bory, Euglena acus, E. oxyuris Schmarda, E. proxima, Gomphonema augur, G. pawulum, Gyrosigma acuminatum (Kützing) Rabenhorst, Hantzschia amphioxys, Melosira varians Agardh, Navicula cuspidata, N. pupula, Nitzschia acicularis, $N$. linearis, $N$. palea, $N$. sigmoidea, Oscillatoria chalybea, Pinnularia maior (Kützing) Rabenhorst y Uva casinoensis Playfair.

El ACP proporcionó sólo diferencias espaciales entre los ríos, así como entre los años 19921993 de 1994-1995, pero no entre épocas contaminadas y no contaminadas, observándose en general, durante todo el período estudiado, un predominio de especies tolerantes. Así pues, el efecto de la contaminación sobre la comunidad fitoplanctónica perduró a lo largo del año, sin lograr una recuperación, a pesar de que químicamente el agua mostró una cierta mejoría. La semejanza en la composición de la ficoflora entre los ríos Salí y Gastona y entre Medina y Marapa puede explicarse, probablemente, por la diferencia de los caudales (Díaz Rueda, 1988) y por la proximidad de sus desembocaduras. Si bien no existe una clasificación regional de los ríos en base al fitoplancton, el Medina y el Marapa se caracterizarían por el predominio de algas potamoplanctónicas (volvocales, euglénidos y diatomeas céntricas) como los de corriente lenta, mientras que el Salí y el Gastona, de corriente rápida, con predominio de Bacillariophyceae pennadas, derivadas del perifiton y bentos, entre otras comunidades (Descy, 1987).

\section{AGRADECIMIENTOS}

Este trabajo ha sido financiado por el Consejo de Investigaciones de la Universidad Nacional de Tucumán (CIUNT), proyecto 26/G128 y Consejo Nacional de Investigaciones Científicas y Técnicas (CONICET), PIP Nº 0871/98.

\section{BIBLIOGRAFÍA}

APHA. 1992. Standard methodsjor the examination of waters and wastewaters. $18^{\text {th }}$. Ed. Amer. Publ. Health Assoc. Washington.

BONETTO, A. A., Y. ZALOCAR de DOMITROVIC y E. R. VALLEJOS. 1982. Contribución al conocimiento del fitoplancton del Paraná Medio. 1. Ecosur, 9 (18): 189-212.

BONETTO, A. A., Y. ZALOCAR de DOMITROVIC y E. R. VALLEJOS. 1983. Fitoplancton y producción primaria del Alto Paraná. Physis, 41 (101): 81 93.

del GIORCIO, P. A., A. VINOCUR, R. J. LOMBARDO \& G. TELL. 1991. Progresive changes in the structure and dynamics of the phytoplankton community allong a polution gradient in a lowland river - a multivariate aproach. Hydrobiologia, 224: 129 154.

DESCY, J. P. 1987. Phytoplankton composition and dinamycs in the river Meuse (Belgium). Arch. Hydrobiol. Suppl., 78 (2): 225-245.

DÍAZ RUEDA, O. 1988. Plan de estudios integrados de la cuenca Río Salí-Dulce. Comité de Cuenca Río Sali-Dulce, I: 401 pp., II: planillas y planos.

GARCÍA de EMILIANI, M. O. 1980. Fitoplancton de una laguna del valle aluvial del Paraná Medio ("Los Matadores", Santa Fe, Argentina). I. Estructura y distribución en relación a factores ambientales. Ecología, 4: 127-140. 
GARCÍA de EMILIANI, M. O. 1986. Fitoplancton de los principales cauces y tributarios del valle aluvial del río Paraná (tramo Goya-Diamante), IV: Análisis de componentes principales y factores comunes. Rev. Asoc. Cienc. Nat. Litoral., 17 (1): 51-61.

GARCÍA de EMILIANI, M. O. 1990. Phytoplankton ecology of the Middle Paraná river. Acta Limnol. Bras., 3: 391-417.

GARCÍA de EMILIANI, M. O. 1997. Effects of water level fluctuations on phytoplankton in a river - floodplain lake system (Paraná river - Argentina). Hydrobiologia, 357: 1- 15.

GÓMEZ, N. 1998. Use of epipelic diatoms for evaluation of water quality in the Matanza-Riachuelo river (Argentina), pampean plain river. Wat. Res., 32 (7): 2029-2034.

GÓMEZ, N. 1999. Epipelic diatoms from the Matanza-Riachuelo river (Argentina), a highly polluted basin from the pampean plain: biotic indices and multivariate analysis. Aquatic Ecosystem Health and Management, 53: 2-9.

LANGE-BERTALOT, H. 1979. Pollution and tolerance of diatoms as criterion of water quality estimation. Nova Hedwigia Beiheft, 64: 285-304.

LOBO, E. A., K. KATOH \& Y. ARUGA. 1995. Response of epilithic diatom assemblages to water pollution in rivers in the Tokyo Metropolitan area, Japan. Fresh Biol., 34: 191-204.

LOCASCIO DE MITROVICH, C., A. VILLAGRA DE GAMUNDI, B. TRACANNA, C SEELIGMANN y C. BUTÍ. 1997. Situación actual de la problemática limnológica de los embalses de la provincia de Tucumán (Argentina). Lilloa, 39: 81-93.

LUQUE, M. E. y A. L. MARTINEZ de FABRICIUS. 2000. Ficoflora fitoplanctónica y epilítica del río Piedra Blanca (Córdoba, Argentina). Bol. Soc. Argent. Bot., 35 (1-2): 21-32.

MARGALEF, R. 1958. Information Theory in Ecology. Gen. Syst., 3: 36-71.

MIRANDE, V. y B. C. TRACANNA. 1995. Estudio cualitativo del fitoplancton del embalse Río Hondo (Argentina) I. Cryptogamie, Algol. 16(4): 211-232.

MIRANDE, V., B. C. TRACANNA y C. T. SEELIGMANN. 1996. Fitoplancton superficial del embalse Río Hondo (Noroeste Argentino) I: zona limnética. Actas del IV Congreso Latinoamericano, II Reunión Ibero-Americana, VII Reunión Brasilera de Ficología, Brasil: 138.

MIRANDE, V., N. ROMERO, M. A. BARRIONUEVO, G. S. MEONI, B. NAVARRO, M. C. APELLA
\& B. C. TRACANNA. 1999. Human impact on some limnological characteristics of the Gastona river (Tucumán, Argentina). Acta Limnologica Brasiliensia, 11 (2): 101-110.

O'FARRELL, I. 1993. Phytoplankton ecology and limnology of the Salado river (Buenos Aires, Argentina). Hydrobiologia, 271: 169-178.

O'FARRELL, I. 1994. Comparative analysis of the phytoplankton of fifteen lowland fluvial systems of the river Plate basin (Argentina). Hydrobiologia, 289: 109-117.

O'FARRELL, I. \& I. IZAGUIRRE. 1994. Phytoplankton ecology and limnology of the river Uruguay lower basin (Argentina). Arch. Hydrobiol. / Suppl. 99 (1-2): 155-179.

PANTLE, R. \& H. BUCK. 1955. Die biologisch Überwachung der Gewasser und die Darstellung der Ergebnisse. Gas-u. Wasserfach, 96: 1-64.

ROMERO, N., M. PAEZ y R. CUEVAS. 1994. Evaluación bienal de la contaminación del dique de Río Hondo. Tankay, 1:329-330.

ROMERO, N., M. J. AMOROSO y B. C. TRACANNA. 1997. Estudio de la carga orgánica y bacteriana en el embalse Río Hondo (Tucumán y Santiago del Estero, Argentina). Miscelanea F.M.L., 103: 1-10.

SEELIGMANN, C. T. 1999. Dinámica del fitoplancton del río Salí (Tucumán, Argentina) en relación a la contaminación. Natura Neotropicalis, 30 (1 y 2): 57-66.

SEELIGMANN, C. T., V. MIRANDE y B. C. TRACANNA. 1996. Fitoplancton superficial del embalse Río Hondo (Noroeste Argentino) II: ríos Salí y Gastona. Actas del IV Congreso Latinoamericano, II Reunión Ibero-Americana, VII Reunión Brasileru de Ficología, Brasil 183 pp.

SEELIGMANN, C. T., S. MARTÍNEZ DE MARCO, S. ISASMENDI y B. TRACANNA. 1999. Impacto de la actividad minera sobre la ficoflora. Rev. Bol. de Ecol., 6: 217-227.

SLÁDECEK, V. 1973. System of water quality from the biological point of view. Arch. Hydrobiol., 7: 1218.

TANGORRA, M., L. M. MERCADO, A. RODRIGUEZ CAPITULO y N. GOMEZ. 1998. Evaluación de la calidad ecológica del A"El Gato a partir del estudio del bentos, fitoplancton y variables físico-químicas. Anales XVII Congreso Nacional del Agua. Santa Fe, Argentina: 1-9.

TRACANNA, B. C., V. MIRANDE y C. SEELIGMANN. 1994. Variaciones del fitoplancton superficial del embalse Río Hondo (Tucumán-Santiago del 
Estero, Argentina), en relación a la actividad azucarera. Tankay, 1: 80-82.

TRACANNA, B. C., C. SEELIGMANN y V. MIRANDE. 1996 (a). Floración de algas azules en el embalse Río Hondo (Noroeste Argentino). Actas del IV Congreso Latinoamericano, II Reunión Ibero-Americana, VII Reunión Brasilera de Ficología, Brasil: 199.

TRACANNA, B. C., C. SEELIGMANN y V. MIRANDE. 1996 (b). Estudio comparativo de la comunidad fitoplanctónica de dos embalses del Noroeste Argentino. Rev. Asoc. Cienc. Nat. Litoral, 27 (1): 13-22.

TRACANNA, B., C. T. SEELIGMANN, V. MIRANDE, L. B. de PARRA, M. T. de PLAZA y F. MOLINARI. 1999. Cambios espaciales y temporales del fitoplancton en el embalse Río Hondo (Argentina). Bol. Soc. Argent. Bot., 34 (1-2): 101-105.
TRACANNA, B. C., S. MARTÍNEZ DE MARCO, C. SEELIGMANN, S. ISASMENDI y A. ELIAS. 2000. Distribución vertical del fitoplancton del embalse Río Hondo, Argentina. En soporte magnético, archivo 06050. En: Uso y preservación de los recursos hídricos en los umbrales del siglo XXI. Farías, H. D. et al. (eds.): 1-7. Memorias del XVIII Congreso Nacional del Agua, Santiago del Estero.

ZALOCAR de DOMITROVIC, Y. 1990. Efecto de las fluctuaciones del nivel hidrométrico sobre el fitoplancton en tres lagunas isleñas en el área de confluencia de los ríos Paraná y Paraguay. Ecosur, 16 (27): 13-29.

ZALOCAR de DOMITROVIC, Y. y E. R. VALLEJOS. 1982. Fitoplancton del río Alto Paraná. Variación estacional y distribución en relación a factores ambientales. Ecosur, 9 (17): 1-28. 